\title{
Simple transmission measurements discriminate instability processes in multiple emulsions
}

\author{
Romaric R. R. Vincent, ${ }^{a}$ Graeme Gillies ${ }^{a b}$ and Anna Stradner ${ }^{* a}$
}

\begin{abstract}
Water-in-oil-in-water double emulsions (W1/O/W2) consist of water droplets (W1) dispersed in oil globules $(\mathrm{O})$, which are then redispersed in an external aqueous phase (W2). This paper reports multiple light scattering investigations on $\mathrm{W} 1 / \mathrm{O} / \mathrm{W} 2$ double emulsions, the inner $\mathrm{W} 1 / \mathrm{O}$ emulsion being a concentrated micron sized inverse emulsion, and the outer $\mathrm{O} / \mathrm{W} 2$ emulsion being a dense direct emulsion. Double emulsions are inherently unstable, and changes of structure can arise from many processes such as instability of both the $\mathrm{W} 1 / \mathrm{O}$ and the $\mathrm{O} / \mathrm{W} 2$ emulsions, and an exchange of water in between the two aqueous phases. Diffuse Transmission Spectroscopy (DTS) is used to follow the structural evolution of these complex systems. We show here that DTS can discriminate between some of the different instability processes of double emulsions. We design three different systems where one of the destabilization processes is dominating, and build different models accounting for the temporal evolution of the turbidity of the system. We find that these models scale well with the experimental data.
\end{abstract}

\section{Introduction}

An emulsion is a mixture of two immiscible liquids, where one liquid (the dispersed phase) is dispersed in the second liquid, the continuous phase. Simple emulsions can be of two kinds: oil-inwater $(\mathrm{O} / \mathrm{W})$ emulsions which are made of oil droplets dispersed in water, while water-in-oil (W/O) emulsions are water droplets dispersed in oil. A double emulsion of water-in-oil-in-water (W1/ $\mathrm{O} / \mathrm{W} 2$ ) is a direct $\mathrm{O} / \mathrm{W} 2$ emulsion, with the dispersed oil phase being made of an inverse $\mathrm{W} 1 / \mathrm{O}$ emulsion. W1 is called the internal or inner aqueous phase while $\mathrm{W} 2$ is the external or outer aqueous phase. Furthermore, double emulsions can be more generally described as of Type-A when a single inner droplet is present in one of the outer droplets, as of Type-B when the coarse droplets contain several small inner droplets, or as Type-C when a large number of inner droplets are present in every coarse outer droplet. In the present study, we will focus on $\mathrm{W} 1 / \mathrm{O} / \mathrm{W} 2$ systems of Type-C, the primary W1/O emulsion being of the concentrated type, and the O/W2 one being of the dense type, with deformed oil droplets.

Double emulsions have potentially a broad range of applications, used as drug carriers in the pharmaceutical industry, and for controlled release of nutrients or flavours in food products. ${ }^{1,2}$

Simple emulsions are metastable systems, and their stability requires the use of a surfactant, the latter being characterised by its hydrophilic-lipophilic balance (HLB). According to

${ }^{a}$ University of Fribourg, Adolphe Merkle Institute, Route de l'ancienne Papeterie, P.O. box 209, 1723 Marly 1, Switzerland. E-mail: anna. stradner@unifr.ch; Fax: +41 26300 9624; Tel: +41 263009120

${ }^{b}$ Fonterra Research Center, Palmerston North, New Zealand(current address)
Bancroft's rule, ${ }^{3}$ high and low HLB surfactants will be used respectively for stabilization of direct and inverse emulsions. For double emulsions, two different surfactants are generally used to stabilize both the primary $\mathrm{W} 1 / \mathrm{O}$ emulsion and the outer $\mathrm{O} / \mathrm{W} 2$ emulsion. Thus double emulsions have two interfaces with opposite properties, and subsequently are highly metastable systems.

From a structural point of view, loss of stability can arise from different aspects. First, both the inner and outer emulsions can be unstable, following processes such as coalescence or Ostwald ripening (formation of larger droplets), creaming (phase separation), or flocculation (aggregation). But added to the simple emulsions case, the osmotic balance between the two aqueous phases plays a major role in the stability, ${ }^{4-6}$ leading to a water exchange from the outer aqueous phase towards the inner phase (swelling), or from the inner phase towards the outer one (water leakage). This obviously leads to important changes of the microstructure, and of the rheology in the case of a dense outer emulsion. In this study, the outer emulsion is of the dense type, the oil droplets are highly deformed and the elasticity of the double emulsion is determined by the elasticity of the interfaces between the droplets. ${ }^{7}$ Thus following the evolution of the elasticity of a double emulsion should help to quantify the volume fraction of the outer aqueous phase $\Phi_{\text {outer }}$, and subsequently a change of $\Phi_{\text {outer }}$ will be linked to water exchanges between the respective aqueous phases, swelling of the oil globules if $\Delta \Phi_{\text {outer }}$ $<0$ or water leakage if $\Delta \Phi_{\text {outer }}>0$.

In order to get insight in how the complex structure of multiple emulsions is evolving, one needs a non-invasive technique adapted to the different scales present in the system. In the present study, the inner droplets are micron-scaled while the 
outer $\mathrm{O} / \mathrm{W} 2$ emulsion has oil droplets of about $10 \mu \mathrm{m}$, which makes light scattering techniques suitable. The structural evolution of dilute double emulsions has been monitored by small-angle light scattering, ${ }^{6}$ and by dynamic light scattering. ${ }^{8}$ One could also perform light scattering on concentrated systems by matching the refractive indexes of the dispersed phase and the continuous phase of a simple emulsion, ${ }^{9}$ but this condition would be rather difficult to achieve for multiple emulsions. Due to the high turbidity of the concentrated emulsions in the present study, Diffusing Wave Spectroscopy (DWS) would be a very relevant technique to study the dynamics in these systems. Michaud et al. ${ }^{10}$ performed DWS on multiple emulsions, and interpreted their results in terms of interactions between the inner droplets and the surface of the globules.

However, we believe that a quantitative analysis of DWS applied to multiple emulsions is extremely challenging as different scattering entities undergoing different dynamics are present. Any quantitative modeling of DWS in such systems thus requires first of all a good understanding of the turbidity and how it is linked to the structure of multiple emulsions. It is the aim of the present work to put this understanding forward. We used a technique called Diffuse Transmission Spectroscopy (DTS), ${ }^{11}$ which we can look at as the multiple scattering analogue of static light scattering, to study the structural evolution of highly scattering samples such as concentrated multiple emulsions. The details of the application of this technique to complex systems such as double emulsions will be given in the experimental part.

We propose here to follow the different processes of instability with a DTS study, which gives insights on the time evolution of the structure of both the inner and outer emulsions. We design systems where one of the destabilization processes is dominating, and build different models accounting for the temporal evolution of the turbidity of the system. We show that these models scale reasonably well with the experimental data, which proves that DTS offers a good way of following the structural evolution of complex turbid systems.

\section{Experimental}

\subsection{Materials}

PGPR (polyglycerol polyricinoleate), vinegar and sunflower oil were provided by Nestlé World trade corporation (8619.01). Salt, sugar and eggs were purchased in a local supermarket. A stock solution of $2 \mathrm{wt} \%$ PGPR in sunflower oil was prepared at room temperature, and lower concentrations were obtained by dilution. The inner water solution was prepared by dissolving $10 \mathrm{wt} \%$ of salt and $5 \mathrm{wt} \%$ of vinegar in Milli-q water. Lower concentrations were obtained by dilution. The outer aqueous phase was composed of $40 \mathrm{wt} \%$ egg yolk, $10 \mathrm{wt} \%$ vinegar, $1 \mathrm{wt} \%$ salt, $4 \mathrm{wt} \%$ sugar, and $45 \mathrm{wt} \%$ Milli-q water. The different emulsification steps were made using a Polytron PT2100 (Kinematica, Switzerland) mixer working at different speeds.

A $25 \mathrm{w} / \mathrm{w} \%$ primary water/oil emulsion was prepared by mixing at high speed (19000 or $30000 \mathrm{rpm}) 5 \mathrm{~mL}$ of inner water solution with $15 \mathrm{~mL}$ of oil solution until a temperature of $313 \mathrm{~K}$ was reached, in order to reach a constant energy input during the emulsification process. After cooling down to room temperature,
$15 \mathrm{~mL}$ of primary emulsion was added on top of $5 \mathrm{~mL}$ of outer water solution, and mixed for $20 \mathrm{~s}$ at $11000 \mathrm{rpm}$. The double emulsion sample was immediately loaded in the rheometer or in the transmission cell.

\subsection{Diffuse transmission spectroscopy DTS}

Diffusing Wave Spectroscopy (DWS) is a technique which allows to perform dynamic light scattering experiments on highly turbid samples, and whose analysis is based on a diffusion equation..$^{12,13}$ DWS is nowadays a widely used multiple light scattering technique, having the ability to probe the dynamics of scattering particles over an unrivaled time range. While many applications are found in the visco-elastic characterization of soft materials by probing the motion of embedded tracer particles (microrheology), DWS allows as well the study of the internal dynamics of colloidal dispersions in the concentrated range when the turbidity is too large for classic light scattering techniques.

A home-built DWS setup has been used, ${ }^{14}$ which combines both the two-cell technique ${ }^{15}$ and the echo-technique. ${ }^{16}$ The used laser is a $50 \mathrm{~mW}$ single longitudinal mode diode laser $(\lambda=657 \mathrm{~nm})$ from CrystalLaser. The scattered light can be detected either in transmission or reflection, by a mono-mode fiber, and subsequently analyzed by a single photon counter and a correlator. The setup is designed in order to fulfill all requirements for a correct DWS measurement, in particular for non-ergodic samples.

The main parameter in the DWS analysis is the transport mean free path $l^{*}{ }^{13}$ This analysis is only valid when the turbidity is high, i.e. when the light path of the sample cell $L$ is much larger than $l^{*} . l^{*}$ is generally determined from an experiment on a reference sample made of a well characterized hard sphere dispersion, where $l^{*}$ can be determined by fitting the field correlation function $g_{2}(t),{ }^{13}$ using the known viscosity. Subsequently for future samples $l^{*}$ is obtained by scaling the value obtained for the reference sample, based on the change in transmitted intensity. Indeed, it is known that for non-absorbing slabs of thickness $L$, the transmitted intensity is directly proportional to $\left(l^{*} / L\right) /(1+$ $4 l^{*} / 3 L$ ), so that by measuring the change in transmittance, the change in $l^{*}$ can be calculated.

Thus measuring $l^{*}$ provides important information on the structural evolutions of a transient system, as $l^{*}$ is related to structural properties. Extracting structural information from transmission measurements has been called Diffuse Transmission Spectroscopy (DTS), ${ }^{11}$ and has been used mainly to follow processes such as the gelation of acidified milk, ${ }^{17}$ or of destabilisation of tailored emulsions. ${ }^{18}$ For a suspension of hard spheres, $I^{*}$ is linked to the structural properties intensity via the equation: ${ }^{19}$

$$
l^{*}=k_{0}^{6}\left(\Pi \rho \int^{2 k_{0}} P(q) S(q) q^{3}, d q\right)^{-1},
$$

with $k_{0}$ the wave number, $\rho$ the particle number density, $P(q)$ the $q$ dependent Mie scattering function, ${ }^{20}$ and $S(q)$ the structure factor. For micro-sized concentrated emulsions at concentrations below the glass transition, it is reasonable to treat the micro-sized droplets under study as hard spheres. ${ }^{21,22}$ Thus eqn (1) will be used to estimate the turbidity of the primary $\mathrm{W} 1 / \mathrm{O}$ 
emulsion alone, as well as the turbidity due to the inner water droplets of the double emulsion systems.

At last, as the system of interest is composed of different scattering entities, each entity will have its own light mean free path. The global transport mean free path is given by: ${ }^{13}$

$$
\left(\frac{1}{l_{\text {total }}^{*}}\right)=\sum_{i=1}^{N}\left(\frac{1}{l_{i}^{*}}\right),
$$

where $l_{i}^{*}$ corresponds to the compound $i$.

\subsection{Rheology}

An ARES rheometer from TA Instruments (Delaware, US) was used with a plate-plate geometry of $50 \mathrm{~mm}$ diameter. Sand paper (GRIT 80) was taped to the plates in order to avoid any wall slippage. This was verified by performing small strain frequency sweeps with different gaps, which were giving comparable elastic and viscous moduli.

The evolution of the elastic modulus is a possible way of probing the water exchange between the two aqueous phases. As the outer emulsion is dense, the oil droplets of the O/W2 emulsion are highly deformed, and the elasticity is directly related to the elasticity of the oil membranes, and is linked to the apparent oil volume fraction via the empirical equation: ${ }^{7}$

$$
G^{\prime}=\alpha \frac{\gamma}{R_{d}} \Phi\left(\Phi-\Phi_{c}\right)
$$

with $G^{\prime}$ the elastic modulus, $\Phi$ the oil droplet volume fraction, $\Phi_{c}$ the random close packing volume fraction, $R_{d}$ the droplet radius, $\gamma$ the interfacial tension, and $\alpha$ an empirical constant. This equation could be used to quantify the apparent oil fraction of the $\mathrm{O} / \mathrm{W} 2$ emulsion.

\subsection{Microscopy}

Micrographs of emulsions were obtained using a Leica TCSSP5 confocal microscope in transmission mode without the use of a die. In focus, non-flocculated droplets were then manually discriminated and traced using Corel draw software. The sizes of traced droplets were analysed using imageJ software. Droplet distributions in simple emulsions were established from over one thousand droplets. Droplet distributions in multiple emulsions were typically established from 300 to 500 droplets. The smaller number of droplets counted in multiple emulsions was required in order to maintain a short image acquisition time compared to the time frame of the experiments.

In order to obtain reproducible droplet sizes a constant magnification was used in all micrographs thereby maintaining a constant resolution error. Furthermore both multiple emulsions and simple emulsions were diluted approximately 20 times immediately prior to optical microscopy. Control experiments demonstrated that this dilution did not affect droplet sizes over the time period in which micrographs were acquired. A 100 micron spacer was used to separate the cover glass and microscope slide to prevent any flattening of droplets. Micrographs were obtained from several focal planes to prevent any gravitational bias.

\section{Modelling}

\subsection{Leakage of inner droplets towards the external phase}

We first consider the case of water leakage by fusion of the inner droplets with the outer O/W2 membrane. In other words, one leakage event consists of the loss of one inner water droplet. We consider a $\mathrm{W} 1 / \mathrm{O} / \mathrm{W} 2$ double emulsion system with micron-scaled monodisperse inner droplets, embedded in oil globules of a much larger size (see Fig. 1-a)). The latter forms an O/W2 emulsion which is of the dense type, such that the oil globules are deformed. Furthermore, we assume that both the inner and outer emulsion are stable on their own, neither undergoing coalescence nor Ostwald ripening. The leakage process is modelled as a fast fusion of one inner droplet with the oil globule's O/W2 interface. This phenomenon has been observed by high speed video microscopy in the work of Gonzalez-Ochoa et al., ${ }^{23}$ and was found to be fast, of the order of a few tenths of milliseconds. Thus we will neglect the influence of the fusion stage in the present model. We assume furthermore that the swelling or shrinking processes of the inner droplets due to different osmotic pressure balances reached a steady state. We choose the water leakage rate in a mean field description of the form:

$$
\frac{\partial \Phi_{\text {inner }}(t)}{\partial t}=-K_{L} \Phi_{\text {inner }}(t),
$$

where $\Phi_{\text {inner }}(t)$ is the inner water phase volume fraction and $K_{L}$ a constant called the leakage rate. The solution of eqn (4) is:

$$
\Phi_{\text {inner }}(t)=\Phi_{\text {inner } 0} \exp \left(-K_{L} t\right)
$$

with $\Phi_{\text {inner }_{0}}$ the initial inner aqueous phase volume fraction. As a matter of fact, we assume that there is conservation of the total amount of water in the system, which gives:

$$
\frac{\partial \Phi_{\text {inner }}}{\partial t}=-\frac{\partial \Phi_{\text {outer }}}{\partial t}
$$

A double emulsion system is composed of two distinct scattering entities, the inner droplets and the oil globules' membranes with respective transport mean free paths $l_{\text {inner }}^{*}$ and $l_{\text {outer }}^{*}$. From eqn (2), the transport mean free path of the double emulsion $l_{\text {double }}^{*}$ is determined by:

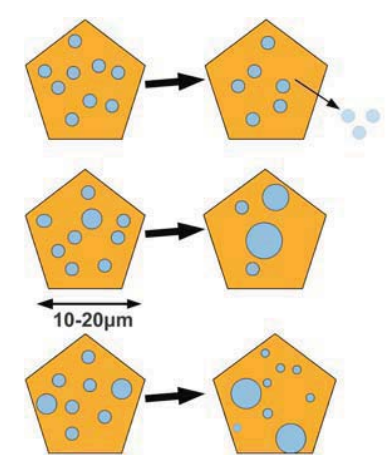

a) Water leakage

b) Coalescence

c) Ostwald ripening

Fig. 1 Schematic of the different instability processes considered in the present paper. a) Water leakage via fusion of the inner aqueous phase droplets toward the outer aqueous phase. b) Coalescence of the inner $\mathrm{W} 1 / \mathrm{O}$ emulsion droplets. c) Ostwald ripening in the inner emulsion. Shown are the deformed oil globules containing the inner water droplets. 


$$
\frac{1}{l_{\text {double }}^{*}}=\frac{1}{l_{\text {inner }}^{*}}+\frac{1}{l_{\text {outer }}^{*}} .
$$

$l_{\text {inner }}^{*}$ can be determined from Mie theory using eqn (1), assuming that the droplets are behaving as hard spheres, which is a reasonable approximation considering the studied internal water volume fraction, around $20 \mathrm{w} / \mathrm{w} \%$ in the present study. From simulations with the appropriate refractive indexes and for a laser wavelength of $657 \mathrm{~nm}$, we find:

$$
l_{\text {inner }}^{*} \simeq \frac{26 R_{\text {inner }}}{\Phi_{\text {inner }}}
$$

where $R_{\text {inner }}$ is the constant inner droplet radius. Eqn (8) is valid only for $R_{\text {inner }}$ ranging from 0.8 to $3 \mu \mathrm{m}$, which is the relevant scale for the studied experimental system.

The case of the oil globule's membrane turbidity is more complicated. We can draw a parallel with the case of dense foams, where the basic ray optics equations rule as the deformed oil globules are much larger than the laser wavelength. There is a fair amount of literature on dense foams, and ray optics simulations have shown that $l_{\text {outer }}^{*}$ scales as: ${ }^{24}$

$$
l_{\text {outer }}^{*} \propto R_{\text {outer }} / \sqrt{\Phi_{\text {outer }}}
$$

with $R_{\text {outer }}$ and $\Phi_{\text {outer }}$ respectively the oil globule radius and outer aqueous phase volume fraction.

If we differentiate eqn (7), we find:

$$
\left(\frac{1}{l_{\text {double }}^{*}}\right)^{2} \frac{\partial l_{\text {double }}^{*}}{\partial t}=\left(\frac{1}{l_{\text {inner }}^{*}}\right)^{2} \frac{\partial l_{\text {inner }}^{*}}{\partial t}+\left(\frac{1}{l_{\text {outer }}^{*}}\right)^{2} \frac{\partial l_{\text {outer }}^{*}}{\partial t} .
$$

This equation is rather complex to solve analytically, and we will neglect the last term. In other words, we choose to neglect the time evolution of the structure of the outer oil membranes. In order to check the validity of this assumption, we estimate the relative values of the different terms of eqn (10) in a 'worst case' scenario: $l^{*}{ }_{\text {inner }}{ }_{0} \simeq 100 \mu \mathrm{m}$ for the initial internal water volume fraction of $20 \mathrm{w} / \mathrm{w} \%$ and a water droplet diameter of $1.8 \mu \mathrm{m}$. Experimentally, for a simple O/W2 egg yolk stabilized emulsion with an oil volume fraction of $75 \mathrm{w} / \mathrm{w} \%$, we find that $l_{\text {outer }}^{*} \simeq$ $200 \mu m$. Using eqn (9) and eqn (10), with $\triangle \Phi_{\text {inner }}=10 \%$ for the total water leakage, we find that the error is about $10 \%$ if the above assumption is made. Thus, we limit the experimental studies to water exchanges of $\triangle \Phi_{\text {inner }} \leq 10 \%$.

Then, using eqn (5) and the simplified eqn (10) leads to the differential equation:

$$
\frac{\partial}{\partial t} \frac{1}{l_{\text {double }}^{*}}=\frac{\Phi_{\text {inner } 0} K_{L}}{26 R_{\text {inner }}}\left(1-\exp \left(-K_{L} t\right)\right) .
$$

This equation has one solution which respects the boundary condition $l_{\text {double }}^{*}(t=0)=l_{\text {double }}^{*}$ :

$$
\frac{1}{l_{\text {double }}^{*}}-\frac{1}{l_{\text {double }_{0}}^{*}}=\frac{1}{l_{\text {inner }_{0}}^{*}}\left(\exp \left(-K_{L} t\right)-1\right),
$$

with $l^{*}{ }_{\text {inner }}=26 R_{\text {inner } 0} / \Phi_{\text {inner } 0}$ the initial transport mean free path linked to the turbidity of the inner emulsion.

\subsection{Coalescence of the inner emulsion}

We now consider a regime where the inner emulsion is in the pure coalescence regime, where neither leakage nor Ostwald ripening take place, as depicted in Fig. 1-b). Again, the inner water droplets are assumed to be much smaller than the oil globules. A simple mean-field description, only valid at the early stage of coalescence, is used: ${ }^{25}$

$$
\frac{\partial n}{\partial t}=-\frac{4 \omega n \pi R_{\text {inner }}^{3}}{3}
$$

where $n$ is the number density of drops of radius $R_{\text {inner }}$, and $\omega$ the coalescence frequency, taken proportional to the droplets volume, which writes as:

$$
\omega(t)=-K_{C} n
$$

with $K_{C}$ a constant called the coalescence rate. Combining eqn (13) and eqn (14) leads to the following differential equation:

$$
\frac{\partial R_{\text {inner }}}{\partial t}=K_{C} \Phi_{\text {inner }} R_{\text {inner }} \text {. }
$$

The solution of this equation respecting the boundary condition $R_{\text {inner }}(t=0)=R_{\text {inner }_{0}}$ is:

$$
R_{\text {inner }}(t)=R_{\text {inner } 0} \exp \left(K_{C} \Phi_{\text {inner }} t\right) .
$$

Then, we use the same formalism as in the case of the water leakage model, using Mie scattering theory to account for the turbidity of the inner droplets (eqn (8)). In eqn (10), the term linked to the turbidity of the outer water/oil interface cancels out since there is no change of the outer aqueous phase fraction. Solving this equation with the limit condition $l_{\text {double }}^{*}(t=0)=$ $l_{\text {double }}$ gives:

$$
\frac{1}{l_{\text {double }}^{*}}-\frac{1}{l_{\text {double }_{0}}^{*}}=\frac{1}{l_{\text {inner }_{0}}^{*}}\left(\exp \left(-K_{C} \Phi_{\text {inner }} t\right)-1\right) .
$$

Interestingly, the time dependence of $l_{\text {double }}^{*}$ has the same scaling as for the water leakage model, its time evolution described by an exponential. This one can rationalize with the fact that coalescence is dominating in both cases: coalescence of the inner emulsion droplets for eqn (17), and coalescence of inner droplets with the oil globule membranes for eqn (12). In the latter case, the inner droplet diameter stays constant while their number density decreases. In the case of coalescence inside the inner emulsion, in the early stages, a decrease of droplet number density will occur while the droplet size will remain relatively constant. So structurally speaking, coalescence of the inner emulsion is similar to water leakage by loss of entire inner droplets. Thus, finding the same scalings with time for the evolution of $l^{*}$ appears reasonable.

We can show that the turbidity of a simple W/O emulsion in the coalescence regime evolves as:

$$
l_{\text {primary }}^{*}=l_{\text {primary }}^{*} \exp \left(K_{C} \Phi_{\text {inner }} t\right) .
$$

\subsection{Ostwald ripening of the inner emulsion}

Finally, we consider the case where the inner emulsion evolves due to Ostwald ripening, and assume there is neither water leakage nor coalescence of the inner emulsion, as depicted in Fig. 1-c). In the case of Ostwald ripening, the theory of 
Lifshiftz-Slyozov-Wagner (LSW) predicts a linear variation of the cube of the average radius of a dispersion as a function of time $^{26,27} R_{\text {inner }}^{3} \propto t$. The LSW theory predicts furthermore a cutoff limit of $1.5 R_{\text {inner } 0}$, and thus is limited to the early stages. In this limit, the scaling $R_{\text {inner }}^{3} \propto t$ has been validated as a signature of Ostwald ripening, ${ }^{25,28,29}$ and the temporal evolution of the droplet radius can be modelled as:

$$
R_{\text {inner }}(t)=R_{\text {inner }_{0}}\left(1+\left(K_{O R} t\right)^{1} / 3\right)
$$

with $K_{O R}$ a constant linked to the Ostwald ripening rate. Similarly to the coalescence regime, we use eqn (10) where the outer term cancels out. The solution of this equation combined with eqn (19) with the boundary condition $l_{\text {double }}^{*}(t=0)=l^{*}{ }_{\text {double }_{0}}$ is:

$$
\frac{1}{l_{\text {double }}^{*}}-\frac{1}{l_{\text {double }_{0}}^{*}}=\frac{1}{l_{\text {inner }_{0}}^{*}}\left[\frac{1}{1+\left(K_{O R} t\right)^{1 / 3}}-1\right] .
$$

The scaling is different than for the previous cases of water leakage and inner droplet coalescence.

Furthermore, we can show that the turbidity of a simple W/O emulsion in the Ostwald ripening regime writes:

$$
l_{\text {primary }}{ }^{*}-l_{\text {primary }}{ }^{*}=l_{\text {primary } 0}{ }^{*}\left(K_{O R} t\right)^{1 / 3} 3
$$

Accordingly, a time dependence of the transport mean free path following a power law of $1 / 3$ appears to be a signature of the Ostwald ripening regime, similarly to what has been concluded in Urbibna-Villalba et al. ${ }^{29}$ for the time dependence of the droplet diameter. This different scaling should allow to distinguish Ostwald ripening from the previous models.

\section{Results and discussion}

\subsection{Initial characterization}

In Fig. 2, the primary W1/O emulsion droplet diameter found via transmission measurements is plotted against the PGPR concentration in the oil phase. The measurements were made one day after the emulsion preparation, which was sufficient to reach a steady state in the transmission. The diameters were

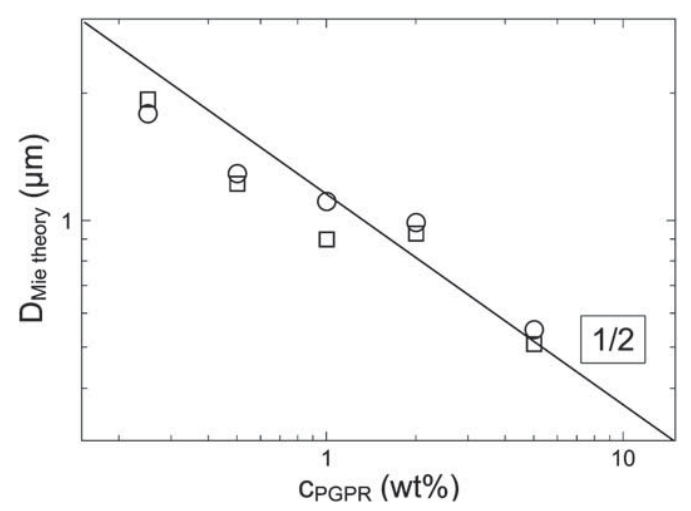

Fig. 2 Primary W1/O emulsion droplet diameter determined from Mie theory using eqn (1), at different PGPR concentrations in the oil phase. The internal water volume fraction is $25 \mathrm{w} / \mathrm{w} \%$. The used mixer speeds are 19000rpm (circles) and 30000rpm (squares). The black line is an arbitrary power law of $1 / 2$. determined by Mie theory simulations for suspensions of homogeneous hard spheres. Interestingly, the diameter appears to depend only on the PGPR concentration, the used mixer speed playing only a minor role. This effect of the PGPR concentration on the W1/O droplet diameters has been reported for PGPR stabilized emulsions in Su et al. ${ }^{30}$ and recently in Marquez et al. ${ }^{31}$ As well, $D_{\text {Mietheory }}$ scales roughly with a power law of $-1 / 2$. If a simple argument of surface coverage by the surfactant was ruling the droplet diameter, $D_{\text {Mietheory }}$ should scale linearly with $c_{P G P R}$, which is clearly not the case. We hypothesize that the surfactant saturation concentration varies with the droplet diameter.

Fig. 3 shows the dependence of both $l^{*}$ and $G^{\prime}$ of a dense O/W2 emulsion on the PGPR concentration in the oil phase, at a constant oil volume fraction of $75 \mathrm{w} / \mathrm{w} \%$. Strikingly, the elastic modulus decreases by a factor of about 5 to finally reach a plateau at $10 \mathrm{~Pa}$ for PGPR concentrations higher than $0.5 \mathrm{wt} \%$. Considering eqn (3), there are two possibilities: either the radius of the oil droplets is changed, or the PGPR surfactant alters the interfacial tension. In Fig. 3, $l^{*}$ changes only slightly, by less than $10 \%$, which is about the uncertainty of the measurement. From eqn (9), we deduce that the oil globule radius does not change markedly, and subsequently that the decrease in elasticity must be linked to a decrease of interfacial tension. The surfactant present in the oil phase is probably adsorbing at the $\mathrm{O} / \mathrm{W}$ interface, as concluded in Michaud et al., ${ }^{32}$ which decreases the interfacial tension. As an interesting remark, the large decrease of $G^{\prime}$ is an indication that the egg yolk stabilized $\mathrm{O} / \mathrm{W}$ emulsions might be stabilized via a pickering process, the pickering particles being some of the proteins or protein aggregates present in the egg yolk. Indeed, pickering emulsions show much higher interfacial tensions than their surfactant-stabilized counterparts, and adding a surfactant to a pickering emulsion will decrease the interfacial tension.

The results depicted in Fig. 3 indicate that rheology results for double emulsions should be treated with caution. Indeed, there would be a competition for the surfactant present in the oil phase between adsorbing at the inner $\mathrm{W} 1 / \mathrm{O}$ interface and adsorbing at the $\mathrm{O} / \mathrm{W} 2$ interface. The influence of the latter process on the

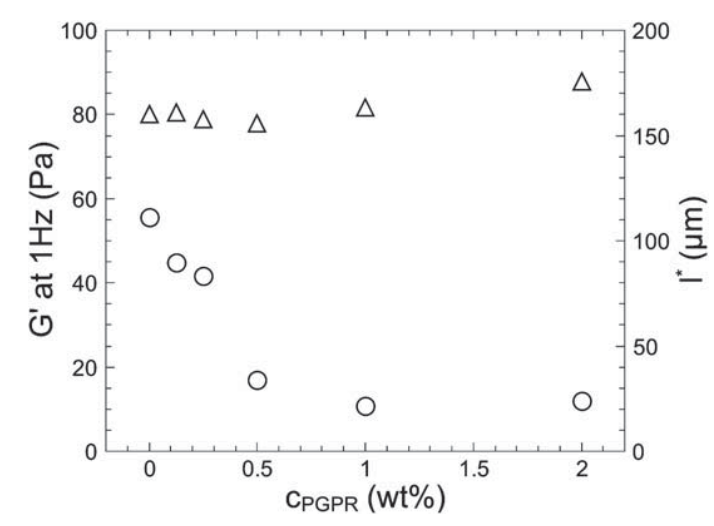

Fig. 3 Elastic modulus (circles) and transport mean free path (triangles) for O/W2 compressed emulsions, at different PGPR concentrations in the oil phase. The oil volume fraction is $75 \mathrm{w} / \mathrm{w} \%$. Small amplitude oscillations $(0.5 \%$ strain) at $1 \mathrm{~Hz}$ were applied. 
rheology is unknown, especially for systems with low to intermediate surfactant concentrations as used in the present paper.

\subsection{Ostwald ripening and coalescence in the primary emulsion}

First, we checked the validity of the Ostwald ripening model (eqn (21)) on simple W1/O emulsions, as shown in Fig. 4-a. For both PGPR concentrations, the transport mean free path evolution scales well with what expected from eqn (21), i.e. a power law of $t^{1 / 3}$. The initial transport mean free path is chosen rather arbitrarily since the actual starting diameter is unknown. Indeed, we need a couple of minutes for the sample transfer into the transmission cell, while the systems evolve rapidly due to Ostwald ripening in the case of simple emulsions. Thus, we will speak mainly about scalings in the rest of the paper as all the descriptive equations are taking into account the initial states.

As explained in detail in Schmitt and Leal-Calderon, ${ }^{25}$ coalescence happens after the Ostwald ripening regime. Eqn (18) is only valid at the early stages of coalescence, when the polydispersity is still reasonably low. Thus, it will be exhibited for primary emulsion systems with more surfactant, where the coalescence regime will be dominating at longer time. The coalescence regime is shown in Fig. 4-b, for a PGPR concentration of $0.5 \mathrm{wt} \%$ in the oil phase. The model related to eqn (18) fits well with the experimental data, up to a certain time when the system may seemingly leave the "early stages" state.

\subsection{Double emulsion systems}

As a preliminary remark, all the double emulsion systems studied in the rest of the paper are supposed to have a stable outer emulsion, i.e. there is neither coarsening nor creaming of the dense $\mathrm{O} / \mathrm{W} 2$ emulsion. This is an adequate assumption since the dynamics of these compressed oil globules is considerably slowed down due to the very high packing. Experimentally, simple O/W2 emulsions stabilized by the same egg yolk solution as used for the double emulsion systems were stable over a very long time. Moreover, we never observed any visible signs of instability of these simple emulsions.

For all the double emulsion samples prepared in the present study, we checked that the swelling was occurring quickly within
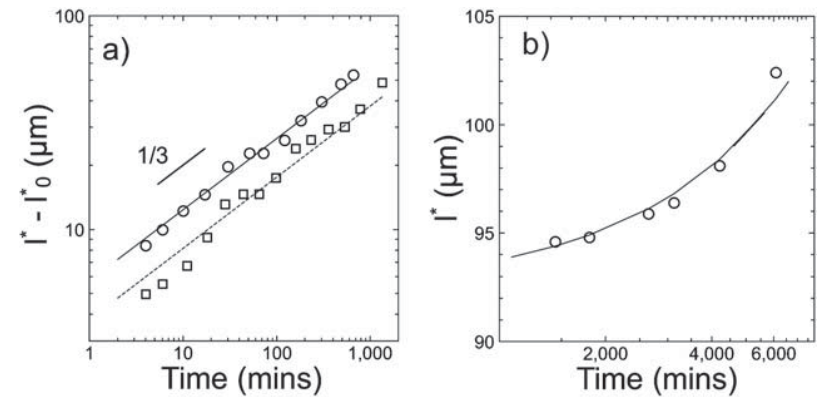

Fig. 4 a) Time evolution of the turbidity of simple W1/O emulsions, consisting of $25 \mathrm{w} / \mathrm{w} \%$ water volume fraction, with $0.125 \mathrm{wt}^{\mathrm{O}} \%$ (circles) and $0.25 \mathrm{wt} \%$ (squares) of PGPR dispersed in the oil phase. The lines are fits from eqn (19). b) Time evolution of the turbidity of a primary W1/O emulsion with a concentration of $0.5 \mathrm{wt} \%$ of PGPR, and an inner aqueous phase volume fraction of $25 \mathrm{w} / \mathrm{w} \%$. The black line is a fit from eqn (18). less than 5 min by following the elastic modulus with rheology (time sweeps). Therefore, in the experimental section, all the transmission experiments are starting at least $5 \mathrm{~min}$ after the sample preparation.

At first, we checked the validity of the model considering the leakage of inner droplets towards the outer aqueous phase, related to eqn (17). In order to limit the effect of coalescence and Ostwald ripening of the inner emulsion, a concentration of $2 \mathrm{wt} \%$ PGPR in the oil phase was used, a concentration sufficiently high to provide a long term stability of the inner water droplets. ${ }^{33}$ However, in order to favor the water leakage, the quantity of salt added to the inner aqueous phase was small, so that the osmotic gradient between the two aqueous phases after the swelling or shrinking process remains limited.

In the two different systems analyzed in Fig. 5, the initial $\mathrm{NaCl}$ concentration differences between the inner and outer aqueous phases are small enough to allow the full osmotic pressure equilibration by swelling or shrinking of the inner water droplets. Under these conditions, the osmotic gradient will be set by the Laplace pressure of the inner droplets, a case studied and modelled in Mezzenga et al. ${ }^{6}$

In the present study, the osmotic balance $\triangle \Psi$ is controlled by $\mathrm{NaCl}$, and the osmotic pressure difference is determined by the Van't Hoff equation, and equal to the Laplace pressure of the inner droplets: ${ }^{6}$

$$
\Delta \Psi=-\Delta[\mathrm{NaCl}]^{*} 2 * R^{*} T=2 * \gamma / r_{\text {inner }} .
$$

where $\Delta[\mathrm{NaCl}]$ is the difference of salt concentration between the 2 aqueous phases, $R^{*} T$ the thermal energy, $\gamma$ the interfacial tension of the internal interfaces, and $r_{\text {inner }}$ the radius of the inner droplets. We can roughly estimate by using the interfacial tension found in $\mathrm{Marze}^{33}$ (PGPR concentration of $2 \% \mathrm{wt}$ in the oil phase,

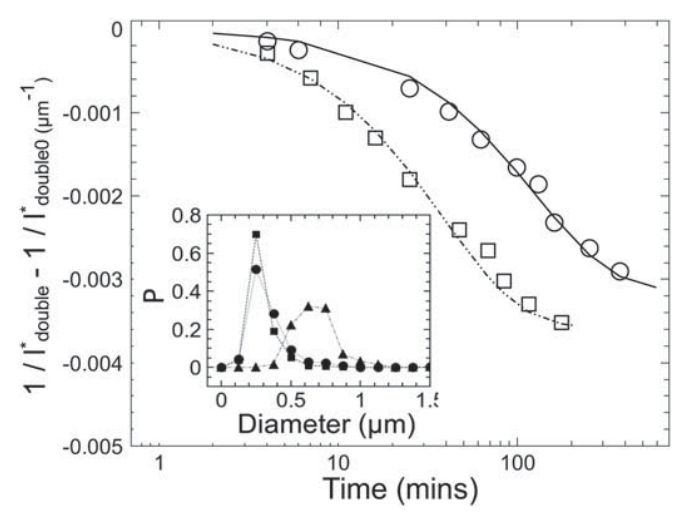

Fig. 5 Time evolution of the turbidity of a double emulsion with a concentration of $2 \mathrm{wt} \%$ of PGPR in the oil phase, an inner aqueous phase volume fraction of $19 \mathrm{w} / \mathrm{w} \%$, and $75 \mathrm{w} / \mathrm{w} \%$ of apparent oil fraction. The salt concentrations in the inner aqueous phase are $0 \mathrm{wt} \%$ (squares) and $2 \mathrm{wt} \%$ (circles). The black continuous and dotted lines are fits from eqn (12). Inset: Time evolution of the inner droplet diameter distribution as determined by confocal microscopy, for a double emulsion system with a concentration of $2 \mathrm{wt} \%$ of PGPR in the oil phase, an inner aqueous phase volume fraction of $19 \mathrm{w} / \mathrm{w} \%$, and $75 \mathrm{w} / \mathrm{w} \%$ of apparent oil fraction. The inner salt concentration is $2 \mathrm{wt} \%$ in the inner aqueous phase. The measurements were performed $110 \mathrm{~min}$ (squares), $600 \mathrm{~min}$ (circles), and $1500 \mathrm{~min}$ (triangles) after the sample preparation. 
$\gamma=2 \mathrm{mM} / \mathrm{m}$ ) and a droplet radius of $1 \mu \mathrm{m}$ that the equilibrium will be found at $\Delta[\mathrm{NaCl}]=0.0049 \mathrm{Mol} / \mathrm{l}=0.28 \mathrm{~g} / \mathrm{l}=0.028 \%$ wt . This small difference of $\mathrm{NaCl}$ concentration will remain after equilibration, and will result in a small positive or negative osmotic gradient in between the two aqueous phases.

In the inset of Fig. 5, the particle size distribution obtained from microscopy barely evolves during the first $600 \mathrm{~min}$, confirming the absence of coarsening of the inner emulsion. After 1500 min a coarsening occurs, and thus we limit the transmission study to about $600 \mathrm{~min}$ for this system.

The time dependence of the turbidity fits well with an exponential law as modeled by eqn (12), up to a time evolution of $500 \mathrm{~min}$ for the system with $2 \mathrm{wt} \%$ salt in the inner phase, as exhibited in Fig. 5. The used $K_{L}$ parameters for the models are $0.026 \mathrm{~s}^{-1}$ and $0.008 \mathrm{~s}^{-1}$ for salt concentrations in the inner phase of 0 and $2 \mathrm{wt} \%$, respectively. In other words, the system with no salt leaks faster than the one with a small amount of salt in the inner phase, which makes sense since for the system with an initial $\mathrm{NaCl}$ concentration of $0 \mathrm{wt} \%$ in the inner phase, a small positive osmotic gradient from the inner phase toward the external phase will remain, which will drive the inner droplets out of the oil globule, while for the system with an initial $\mathrm{NaCl}$ concentration of $2 \mathrm{wt} \%$ in the inner phase, the remaining opposite osmotic gradient will slow down the leakage of the inner droplets. From this point of view it makes sense that the system without salt leaks faster than the one with a small amount of salt in the inner phase.

In a second step, double emulsion systems were designed such that the Ostwald ripening process is dominating the inner emulsion evolution. $10 \% \mathrm{wt}$ of $\mathrm{NaCl}$ was present initially in the inner aqueous phase, and an equilibrium of osmotic pressure between the two aqueous phases was impossible to reach since the outer phase is of a limited volume. Therefore, after an initial and rapid swelling of the inner droplets, a large osmotic pressure gradient was remaining from the outer phase towards the inner phase, slowing down the leakage of inner droplets towards the outer phase.

The particle size distribution obtained from confocal images is shown in the inset of Fig. 6. It can be seen than over the 210 first minutes, the number of large droplets is increasing while the number of small droplets stays relatively constant. This is in good agreement with the presence of Ostwald ripening. Ideally, a decrease of the size of the small droplets should be seen, but the actual spacial resolution appears not sufficient to see any effects. In Fig. 6, the evolution of the turbidity of double emulsion systems made with the same concentration of PGPR in the oil phase as in Fig. 4 is plotted. For both systems, the fits with eqn (17) are in good agreement, considering again only the scaling. As said previously, the absolute values are very arbitrary as the starting system at $t=0$ is not well defined. Nevertheless, the $K_{O R}$ constants used in the fits in Fig. 6 are at least one order of magnitude larger than the ones used in Fig. 4-a, which could be a further indication that some of the PGPR of the primary emulsion diffuses towards the oil globule membrane. Thus, the effective PGPR concentration in the oil phase is lower than for the case of the simple primary emulsion, which explains why the Ostwald ripening goes faster.

Eventually, similarly to the section concerning simple emulsions, we designed a system where the coalescence of the inner

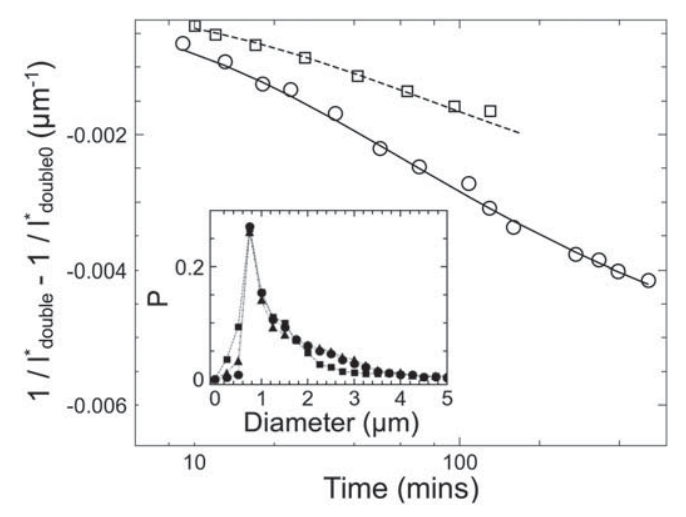

Fig. 6 Time evolution of the turbidity of a double emulsion system with $10 \mathrm{wt} \%$ salt in the inner aqueous phase, $19 \mathrm{w} / \mathrm{w} \%$ as the total internal water phase fraction, and $75 \mathrm{w} / \mathrm{w} \%$ of apparent oil fraction. $0.125 \mathrm{wt} \%$ (squares) and $0.25 \mathrm{wt} \%$ (circles) of PGPR were present in the oil phase. The lines are given by eqn (20) for both systems. Inset: Time evolution of the inner droplet diameter distribution as determined by confocal microscopy, for a system with $10 \mathrm{wt} \%$ salt in the inner aqueous phase, 19 w/w $\%$ as the total internal water phase fraction, and $75 \mathrm{w} / \mathrm{w} \%$ of apparent oil fraction. $0.25 \mathrm{wt} \%$ of PGPR was present in the oil phase. The measurements were performed $20 \mathrm{~min}$ (squares), $80 \mathrm{~min}$ (circles), and $210 \mathrm{~min}$ (triangles) after the sample preparation.

emulsion was dominating. To do so, we increased the PGPR concentration to $1 \mathrm{wt} \%$ in the oil phase, a concentration necessary to get a more slowly evolving system, limiting the structural changes due to Ostwald ripening. In the inset of Fig. 7, the droplet size distribution evolves only very little over the first $1500 \mathrm{~min}$, a sign that the system is still in the early stages of the coalescence regime. After $3000 \mathrm{~min}$, one can see a large increase of the droplet diameter, associated to a broader distribution.

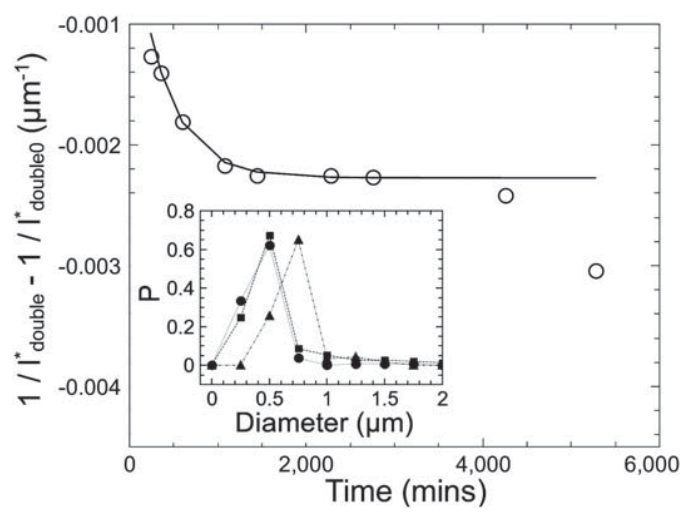

Fig. 7 Time evolution of the turbidity of a double emulsion system with $10 \mathrm{wt} \%$ salt in the inner aqueous phase, $19 \mathrm{w} / \mathrm{w} \%$ as the total internal water phase fraction, and $75 \mathrm{w} / \mathrm{w} \%$ of apparent oil fraction. $1 \mathrm{wt} \%$ (circles) of PGPR was present in the oil phase. The line is a fit of eqn (17). Inset: Time evolution of the inner droplet diameter distribution for a double emulsion system with $10 \mathrm{wt} \%$ salt in the inner aqueous phase, 19 w/w $\%$ as the total internal water phase fraction, and $75 \mathrm{w} / \mathrm{w} \%$ of apparent oil fraction, as determined by confocal microscopy. $1 \mathrm{wt} \%$ (circles) of PGPR was present in the oil phase. The measurements were performed $210 \mathrm{~min}$ (squares), $1500 \mathrm{~min}$ (circles), and $3000 \mathrm{~min}$ (triangles) after the sample preparation. 
Thus, the limit of validity of eqn (17) would be somewhere in between 1500 and $3000 \mathrm{~min}$.

The evolution of the turbidity is shown in Fig. 7. The model for double emulsions with a coalescing inner emulsion (eqn (17)) appears to fit very well with the experimental data, up to about $3000 \mathrm{~min}$ when the turbidity tends to deviate from the model. This is in very good agreement with what was observed by confocal microscopy. This latter effect is expected to be linked to the leakage of inner water droplets. Indeed, it is in this period that a visible destabilisation starts, with a layer of water phase forming at the bottom of the sample cell. This is likely to be due to water leakage of the larger inner water droplets, which increases the outer aqueous phase fraction up to a stage where the $\mathrm{O} / \mathrm{W} 2$ emulsion is not compressed any more, leading to creaming.

\section{Conclusion}

We demonstrated that simple DTS measurements reveal to be a practical tool to study the structural evolution of turbid complex systems, if the appropriate modelling is developed for taking into account the different contributions to turbidity. In the present study, we built three different models accounting for different destabilisation processes of double emulsions, and show experimentally that they describe reasonably well the structural evolution during Ostwald ripening of the inner emulsion, coalescence of the inner emulsion, and leakage of inner water droplets towards the external aqueous phase. It should be possible to build models describing the structural evolution of many other complex turbid systems. The double emulsion systems in this study are not well defined as it is usually the case in industrial studies, but appear to qualitatively scale well with the models. These models would reveal useful to follow emulsions and multiple emulsions in many industrial applications, since the experimental technique can be easily implemented on a production line.

\section{Acknowledgements}

This work was financially supported by the University of Fribourg, the Nestlé Research Center (NRC, Switzerland), and the Adolphe Merkle Foundation (Switzerland). We thank A. Syrbe (NRC) and B. Valles-Pamies (NRC) for interesting discussions and help with sample preparation. Prof. P. Schurtenberger is acknowledged for helful comments and encouragements.

\section{References}

1 N. Garti and C. Brisperink, Curr. Opin. Colloid Interface Sci., 1998, 3, 657.

2 G. Muschiolik, Curr. Opin. Colloid Interface Sci., 2007, 12, 213.

3 W. Bancroft, J. Phys. Chem., 1913, 17, 501.

4 J. Jiao, D. Rhodes and D. Burgess, J. Colloid Interface Sci., 2002, 250, 444.

5 L. Wen and K. Papadopoulos, J. Colloid Interface Sci., 2001, 235, 398.

6 R. Mezzenga, B. Folmer and E. Hughes, Langmuir, 2004, 20, 3574.

7 T. Mason, J. Bibette and D. Weitz, Phys. Rev. Lett., 1995, 75, 2051.

8 W. Yafei, Z. Tao and H. Gang, Langmuir, 2006, 22, 67.

9 H. Lindner, G. Fritz and O. Glatter, J. Colloid Interface Sci., 2001, 242, 239.

10 F. Michaud, P. Hébraud and P. Perrin, Polym. Int., 2003, 52, 594.

11 P. Kaplan, A. Dinsmore, A. Yodh and D. Pine, Phys. Rev. E: Stat. Phys., Plasmas, Fluids, Relat. Interdiscip. Top., 1994, 50, 4827.

12 D. Pine, D. Weitz, P. Chaikin and E. Herbolzheimer, Phys. Rev. Lett., $1988,60,1134$.

13 D. Weitz and D. Pine, Dynamic Light Scattering: The Method and Some Applications, Oxford University Press, 1993.

14 P. Zakharov, F. Cardinaux and F. Scheffold, Phys. Rev. E: Stat., Nonlinear, Soft Matter Phys., 2006, 73, 011413.

15 F. Scheffold, S. Skipetrov, S. Romer and P. Schurtenberger, Phys. Rev. E: Stat. Phys., Plasmas, Fluids, Relat. Interdiscip. Top., 2001, 63, 061404.

16 P. Hébraud, F. Lequeux, J. Munch and D. Pine, Phys. Rev. Lett., 1997, 78, 4657.

17 M. Corredig and M. Alexander, Trends Food Sci. Technol., 2008, 19, 67.

18 H. Ruis, K. van Gruijthuijsen, P. Venema and E. van der Linden, Langmuir, 2007, 23, 1007.

19 S. Fraden and G. Maret, Phys. Rev. Lett., 1990, 65, 512.

20 H. V. de Hulst, Light Scattering by Small Particles, Dover, New York, 1981.

21 M. Gradzielski and H. Hoffmann, Adv. Colloid Interface Sci., 1992, 42, 149 .

22 R. Aboofazelli, D. Barlow and M. Lawrence, AAPS ParmScience, 2000, 2, 13

23 H. Gonzalez-Ochoa, L. Ibarra-Bracamontes and J. Arauz-Lara, Langmuir, 2003, 19, 7837.

24 M. Vera, A. Saint-James and D. Durian, Appl. Opt., 2001, 40, 4210.

25 V. Schmitt and F. Leal-Calderon, Europhys. Lett., 2004, 67, 662.

26 I. Lifshiftz and V. Slyozov, J.Phys. Chem., 1961, 19, 35.

27 Z. Wagner, Z. Electrochem., 1961, 65, 581.

28 S. Egelhaaf, U. Olsson, P. Schurtenberger, J. Morris and H. Wennerström, Phys. Rev. E: Stat. Phys., Plasmas, Fluids, Relat. Interdiscip. Top., 1999, 60, 5681.

29 G. Urbina-Villalba, A. Forgiarini, K. Rahn and A. Lozsan, Phys. Chem. Chem. Phys., 2009, 11, 11184.

30 J. Su, J. Flanagan, Y. Hemar and H. Singh, Food Hydrocolloids, 2006, 20, 261.

31 A. Marquez, A. Medrano, L. Panizollo and J. Wagner, J. Colloid Interface Sci., 2010, 341, 101.

32 F. Michaud, P. Perrin and P. Hébraud, Langmuir, 2004, 20, 8576.

33 S. Marze, Langmuir, 2009, 25, 12066. 